\title{
In tune with nature: Wolbachia does not prevent pre-copula acoustic communication in Aedes aegypti
}

João Silveira Moledo Gesto ${ }^{1,2}$, Alejandra Saori Araki ${ }^{2}$, Eric Pearce Caragata', Caroline Dantas de Oliveira', Ademir Jesus Martins ${ }^{3,4}$, Rafaela Vieira Bruno ${ }^{2,4 \dagger}$ and Luciano Andrade Moreira ${ }^{1,4^{*}+}$ (D)

\begin{abstract}
Background: Mosquito-borne diseases are rapidly spreading to vast territories, putting at risk most of the world's population. A key player in this scenario is Aedes aegypti, a hematophagous species which hosts and transmits viruses causing dengue and other serious illnesses. Since vector control strategies relying only on insecticides have proven unsustainable, an alternative method involving the release of Wolbachia-harboring individuals has emerged. Its successful implementation vastly depends on how fit the released individuals are in the natural habitat, being able to mate with wild populations and to spread Wolbachia to subsequent generations. In mosquitoes, an important aspect of reproductive fitness is the acoustic communication between males and females, which translates to interactions between harmonic frequencies in close proximity flight. This study aimed to characterize the flight tone produced by individuals harboring Wolbachia, also evaluating their ability to establish stable acoustic interactions.
\end{abstract}

Methods: Wild-type (WT) and Wolbachia-harboring specimens (WMelBr) were thorax-tethered to blunt copper wires and placed at close proximity to sensitive microphones. Wing-beat frequencies (WBFs) were characterized at fundamental and harmonic levels, for both single individuals and couples. Harmonic interactions in homogeneous and heterogeneous couples of WT and WMelBr variants were identified, categorized and quantified accordingly.

Results: In tethered 'solo' flights, individuals harboring Wolbachia developed WBFs, differing slightly, in a sex-dependent way, from those of the WT strain. To test the ability to form harmonic 'duets', tethered couples of WMelBr and WT individuals were shuffled in different sex pairs and had their flight tones analyzed. All couple types, with WT and/ or WMelBr individuals, were able to interact acoustically in the frequency range of $1300-1500 \mathrm{~Hz}$, which translates to the convergence between male's second harmonic and female's third. No significant differences were found in the proportions of interacting couples between the pair types. Surprisingly, spectrograms also revealed the convergence between alternative harmonic frequencies, inside and outside the species putative hearing threshold.

Conclusions: Wolbachia infection leads to small sex-dependent changes on the flight tones of Ae. aegypti, but it does not seem to prevent the stereotyped harmonic interaction between males and females. Therefore, when released in the natural habitat to breed with native individuals, Wolbachia-harboring individuals shall be fit enough to meet the criteria of acoustically-related mating behavior and promote bacteria dispersion effectively.

Keywords: Wolbachia, Aedes aegypti, Mating, Bioacoustics, Harmonic convergence, Fitness

\footnotetext{
* Correspondence: luciano@minas.fiocruz.br

${ }^{\dagger}$ Equal contributors

${ }^{1}$ Mosquitos Vetores: Endossimbiontes e Interação Patógeno-Vetor, Instituto

de Pesquisas René Rachou, Fiocruz, Belo Horizonte, MG, Brazil

${ }^{4}$ Instituto Nacional de Ciência e Tecnologia em Entomologia Molecular

(INCT-EM)/CNPq, Rio de Janeiro, Brazil

Full list of author information is available at the end of the article
} 


\section{Background}

Diseases transmitted by mosquito vectors, with an ever growing human burden, pose a real threat to global public health. The hematophagous species Aedes aegypti is a major player in this scenario, being able to host and transmit viruses causing dengue, chikungunya, urban yellow fever and Zika [1-5]. Dengue is the most prevalent arboviral disease, with an estimated 390 million annual infections [1] in over 128 countries [6]. Albeit less frequent, chikungunya and Zika infections are rising and spreading to new territories, including the American continent, where major outbreaks have been reported [7-10]. The remaining burden of vaccine-preventable yellow fever is also alarming and believed to be underestimated in places like Africa, where the annual incidence ranges from 51,000 to 380,000 cases [11].

The escalation of arbovirus infections across the globe is largely attributed to the success of Ae. aegypti as a vector [12]. Due to its highly anthropophilic behavior and the ability to quickly adapt to urban environments [13, 14], this species is invading new territories and augmenting its occurrence $[15,16]$. With no effective vaccines for dengue, chikungunya or Zika, or even therapeutic drugs to alleviate the diseases' symptoms, vector control initiatives are the only solution available to fight epidemic outbreaks. Most of these initiatives combine educational approaches, engaging the population to eliminate breeding sites, and the use of insecticides to suppress mosquito populations [12, 17-20]. However, strategies relying on insecticides have proven ineffective and unsustainable for the long term, due to the surge of resistant populations [21, 22].

Recently, an innovative approach using the endosymbiotic bacterium Wolbachia pipientis has been successfully implemented to control the transmission of arboviruses by Ae. aegypti [23-26]. Naturally present in around $40 \%$ of the arthropods [27], Wolbachia is an obligatory intracellular symbiont, which promotes its own transmission by manipulating host reproduction through a mechanism known as cytoplasmic incompatibility (CI) [28]. Following an artificial introduction of the bacteria into Ae. aegypti [29], a complex host-symbiont association arose and led to an efficient pathogen interference (PI) phenotype, blocking the transmission of dengue, chikungunya and Zika [30, 31].

Fitness costs are also a byproduct of this recent hostsymbiont association, thus representing an important concern to release programs of Wolbachia-infected lines [26, 29, 32-34]. Depending on the combination between host background and Wolbachia strains, higher or lower costs can arise and directly affect the efficacy of which the bacteria spread through native populations [35]. Inducing shorter developmental time and a slightly reduced lifespan, yet keeping strong CI and PI phenotypes, wMel has been the preferred Wolbachia strain for control programs
$[23,25,26]$. However, some fundamental aspects of reproductive fitness, such as mating behavior, have not been yet assessed for this strain.

Acoustic signals produced during flight play an important role in mosquito mating success. Sexual recognition occurs when males and females, flying within hearing distance, adjust their wing-beat frequencies so that harmonic components can interact. While some species simply converge their fundamental frequencies [36], the great majority of the Culicidae, including Ae. aegypti, seem to induce frequency matching at higher harmonics, usually involving male's second and female's third components [37-40]. Most importantly, these interactions seem to be important cues for mating success, influencing females' rejection/ acceptance behaviors toward males [41]. For this reason alone, our understanding of mosquito mating behavior is particularly relevant, and should be in taken into consideration when developing control strategies based on the release of Wolbachia-harboring lines.

In this report, we characterized the wing-beat frequency of Wolbachia-infected Ae. aegypti and evaluated their ability to sexually communicate through acoustic signals. To give this work a sense of field application, we chose $w \mathrm{MelBr}$ as our Wolbachia-hosting strain. wMelBr is currently being utilized by the 'Eliminate Dengue' program in Brazil (http://www.eliminatedengue.com/br) and was obtained by repeated backcrossing $(8 x)$ of the original Australian $w$ Mel strain with a wild-type (WT) population from Rio de Janeiro.

\section{Methods}

Both $w \mathrm{MelBr}$ and control (WT) strains were maintained following a standard protocol. Eggs were randomly selected and hatched in distilled water at $28 \pm 2{ }^{\circ} \mathrm{C}$. Larvae were sorted into trays filled with $1 \mathrm{l}$ of distilled water and fed a diet of Tetramin ${ }^{\odot}$ Tropical Tablets (Tetra, Spectrum Brands, Blacksburg, Virginia, USA). Following emergence, adults were immediately sexed (to avoid insemination) and kept at $25{ }^{\circ} \mathrm{C}$ on a $10 \%$ sucrose diet. Both larvae and adults were reared at 12:12 light-dark cycle.

Experiments were performed during the early morning (ZT0-3; ZT0 = lights ON) and late afternoon hours (around dusk) (ZT9-12; ZT12 = lights OFF), when Ae. aegypti is known to be more active [42]. Individuals from the age range of 6 to 10 days were anesthetized on ice and thorax-tethered with super glue to a blunt copper wire. Next, they were positioned at a $2 \mathrm{~mm}$ distance to a particle velocity sensitive microphone, located inside a recording apparatus known as INSECTAVOX [43], which was originally developed for acquiring signals from Drosophila courtship songs but also proved suitable for mosquito flight tones. Inhibition and stimulation of flight activity was achieved through tarsal contact with a fragment of tissue paper. Recordings of single individuals or couples 
were performed at $25 \pm 1{ }^{\circ} \mathrm{C}$ and monitored in real-time, which allowed us to discard samples with erratic wingbeats. For single individuals, recording time was set to $30 \mathrm{~s}$, while for couples there was no time limit, with flight allowed (and stimulated) more than once per recording.

Microphone voltage output was sampled at $44.1 \mathrm{~Hz}$, 16-bit, using Spectrogram v.16 (Visualization Software LLC, Stafford, Virginia, USA). Sound data was stored in wav files and analyzed with Raven Pro v.1.4 (The Cornell Lab of Ornithology, Cornell University, Ithaca, New York, USA; Available from http://www.birds.cornell.edu/raven). Spectrograms were generated following a discrete Fourier Transform (DFT) (every 4096 points or 92.9 ms), Hanning windowing and $50 \%$ overlap. Harmonics were measured by manually selecting the spectrograms and applying the 'center frequency' algorithm, whose output is the frequency that divides the selection into two frequency intervals of equal energy [44]. Selections spanned $6 \mathrm{~s}$ for 'solo flights' and 1$4 \mathrm{~s}$ for couples with visual indication of convergence (a.k.a. frequency matching), which was corroborated by spectrogram slices with higher resolution. Convergence was not computed, and therefore considered absent in the couple analyzed, if matching frequencies lasted less than $1 \mathrm{~s}$.

\section{Results}

To investigate the effect of Wolbachia on the modulation of flight tones, we measured the wing-beat frequencies of tethered single individuals, randomly selected from $w \mathrm{MelBr}$ and WT control strains (Fig. 1). In 'solo' flight, the fundamental frequencies $(\mathrm{F} 1)$ of $w \mathrm{MelBr}$ males and females were $713.5 \pm 8.2 \mathrm{~Hz}$ and $495.3 \pm 5.1 \mathrm{~Hz}$ (mean \pm SEM), respectively. The WT control strain showed a similar pattern, with $697.6 \pm 7.3 \mathrm{~Hz}$ for males and $513.7 \pm 5 \mathrm{~Hz}$ for females. Statistical analysis of variance (ANOVA) revealed that, while there was a highly significant effect for $\operatorname{sex}\left(F_{(1,131)}=\right.$ 873.4, $P<0.0001)$, there was none for $\operatorname{Wolbachia}\left(F_{(1,131)}=\right.$ $0.03728, P=0.8472)$. Yet, an interesting sex $\times$ Wolbachia interaction arose $\left(F_{(1,131)}=6.353, P=0.0129\right)$, indicating

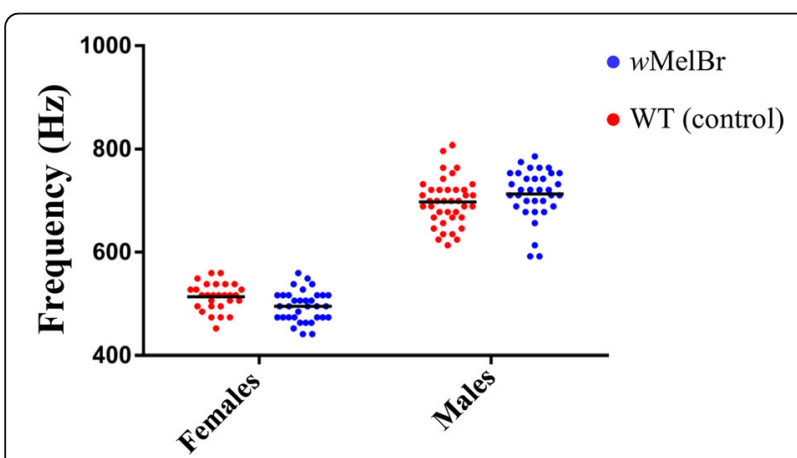

Fig. 1 Wing-beat frequencies of WMelBr and WT control individuals in 'solo' tethered flight. Each dot represents the computed fundamental frequency (i.e. 1st harmonic) for a single adult individual, while the horizontal black lines indicate the mean that the bacteria influence wing-beat frequencies of males and females in opposite ways. Indeed, careful examination of the data plot (Fig. 1) reveals that frequencies for females are slightly lower in Wolbachia-harboring individuals, while those for males are higher. Since males are able to detect and orient towards females' flight tones [45-47], this difference could possibly be an underlying basis for discriminating and selecting sexual partners from each variant. As such, follow-up behaviors were analyzed.

In order to evaluate the effect of Wolbachia on the acoustic communication associated with mating, we measured the wing-beat frequencies of tethered couples flying in close proximity. Spectrograms were carefully analyzed for a 'duet' formation, which occurs when harmonic frequencies from males and females converge to a common frequency band [37]. Initially, our attention focused on the convergence between females' third (F3) and males' second (M2) harmonics, which was found to be an important pre-copula event $[37,41]$. To provide a broader picture of any Wolbachia-driven effect and re-create the different scenario found when infected individuals are released in the wild, all the possible sex pairs (or couple types) between $w \mathrm{MelBr}$ and WT were evaluated. Interestingly, our results revealed that all the combinations were able to interact acoustically, suggesting that Wolbachia does not prevent this phenomenon (Fig. 2). In strictly WT

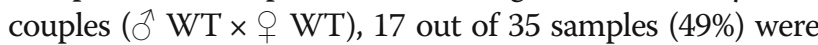
able to form 'duets' and interact at $1441.5 \pm 21.1 \mathrm{~Hz}$, with an interquartile range (IQR) equal to $13.3 \pm 1.7 \mathrm{~Hz}$. In couples formed by WT males and $w$ MelBr females $(\hat{0} \mathrm{WT} \times$ $w \mathrm{MelBr}$ ), duets were observed in 10 out of 28 samples (36\%), with converging frequencies of $1357.7 \pm 15.8 \mathrm{~Hz}$ and IQR of $10.8 \pm 0.0 \mathrm{~Hz}$. As for couples of $w \mathrm{MelBr}$ males and WT females ( 0 welBr $\times$ + $\mathrm{WT}$ ), 6 out 21 samples $(29 \%)$ were found to converge at $1419.4 \pm 34.4 \mathrm{~Hz}$, with IQR of $12.6 \pm 1.8 \mathrm{~Hz}$. At last, in strictly $w \mathrm{MelBr}$ couples ( $\widehat{\delta}$ $w \mathrm{MelBr} \times$ \& $w \mathrm{MelBr}), 17$ out of 32 samples $(53 \%)$ showed harmonic interaction at $1424.4 \pm 17.6 \mathrm{~Hz}$ and IQR equal to $13.9 \pm 1.2 \mathrm{~Hz}$. One-way ANOVA showed no differences between the means of converging samples from each couple type $\left(F_{(3,46)}=2.692, P=0.057\right)$, indicating that interaction between F3 and M2 occurs in similar frequency ranges. To assess the ability of couples to form these duets, and reveal effects driven by Wolbachia, we performed binary logistic regression analyses using SPSS v.17 (IBM). No significant differences were found between strictly WT couples and other types (Wald $\chi^{2}=4.078, d f=3, P=0.253$ ), both in the overall model and in subsequent pairwise comparisons. In addition, it seems that WT males are equally prone to interact with WT or $w$ MelBr females (Wald $\left.\chi^{2}=1.043, d f=1, P=0.307\right)$, and the reciprocal situation seems to be true for WT females (Wald $\chi^{2}=2.123, d f=1$, $P=0.145)$. A similar, non-significant effect, is found when 


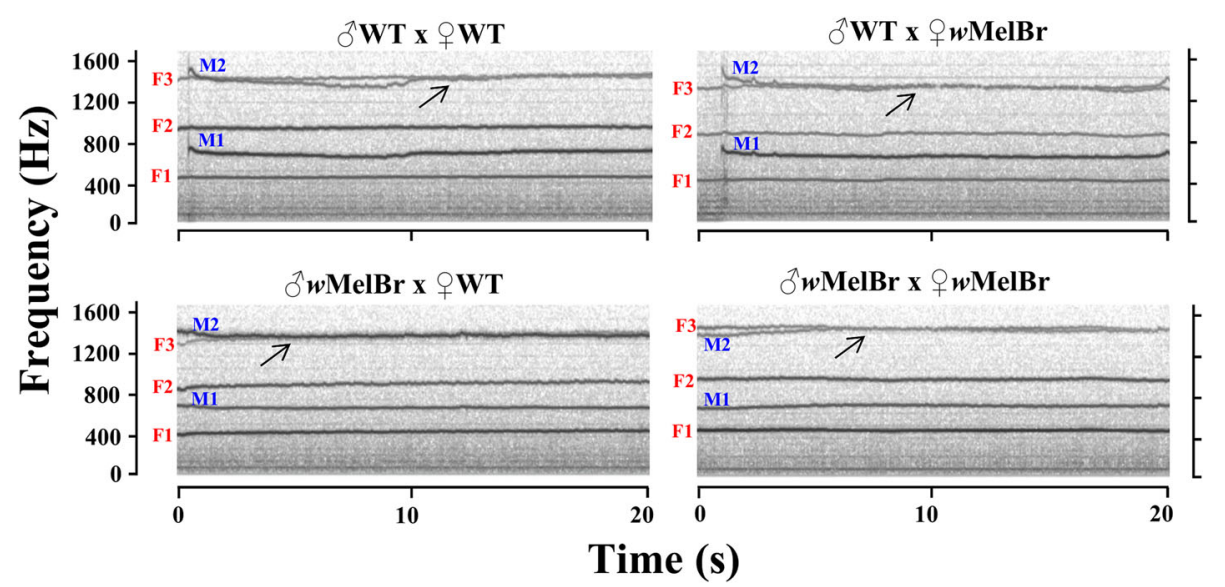

Fig. 2 Wolbachia does not impair convergence between third harmonic frequencies (F3) for females (red) and second (M2) harmonic frequencies for males (blue) of Ae. aegypti. Acoustic interactions were detected in all couple types, formed by WT and/or wMelBr individuals. Arrows indicate converging events (aka. Frequency matching). Horizontal and vertical axes represent time (s) and frequency $(\mathrm{Hz})$

$w \mathrm{MelBr}$ and WT individuals are challenged by $w \mathrm{MelBr}$ males (Wald $\chi^{2}=3.023, d f=1, P=0.082$ ) or females (Wald $\chi^{2}=1.809, d f=1, P=0.179$ ). Ultimately, our statistics suggest that F3/M2 interaction is probably not affected by Wolbachia.

Although most acoustic interactions occurred between F3 and M2, our spectrogram analyses also detected convergence between other harmonic components. In fact, the distribution of all converging samples among couple types revealed a varied array of interactions (Fig. 3a). A fairly common event, for instance, was the interaction between F4 and M3 harmonics (Additional file 1: Figure S1). Less frequent ones included F1/M1, F2/ M1, F5/M3, F5/M4 and F6/M4. In terms of percentage, alternative convergence contributes considerably to interaction indexes between couples (Fig. 3b), which could suggest a functional yet unrevealed role. To provide additional insights on the relative contribution of alternative interactions, new statistical analyses were carried out with data sorted in three categories (i.e. 'F3/M2', 'Other' and 'No interaction'). No significant effect was found between couple types $\left(\chi^{2}=10.49, d f=6, P=0.1056\right)$, which was further corroborated by pairwise comparisons with multiple corrections assuming a false discovery rate of 0.05 . Thus, as it was previously observed, it seems that all couples types show roughly the same ability to interact, even if we consider alternative convergence as a separate category. Another observation that can be drawn from the distribution of converging samples (Fig. $3 \mathrm{a}$ ) is that heterogeneous combinations (i.e. mixed couples) seem to produce more dispersed data (higher standard deviation) than homogeneous ones (Additional file 2: Table S1). This is particularly evident in couples formed by $w \mathrm{MelBr}$ males and WT females (SD $=650.7)$, which should lead to CI and infertile female and, therefore, no offspring. One could speculate that heterogeneous couples, despite being equally able to interact, find some degree of difficulty. Future assays with larger sample sizes, and conditions that better mimic natural mating behavior (e.g. free-flying samples), would be necessary to confirm this effect and take any further conclusions with regards to its functional significance.

\section{Discussion}

By characterizing the wing-beat frequencies of Wolbachiaharboring mosquitoes, as well as identifying and analyzing putative acoustic interactions, this work provided novel and important data on the mating behavior of Ae. aegypti. First, we revealed that the bacteria affect the wing-beat frequencies of individuals flying 'solo', in a sex-dependent fashion. This frequency modulation could be driven by the physical presence of the bacteria either in some sensory organs like the antenna or in flight muscles that mechanically drive wing-beats [23]. Second, we demonstrated that Wolbachia does not prevent couples from interacting acoustically by converging harmonic components. As expected, the most common interaction was that between males' second and females' third harmonics, forming a well-document duet related to mating success [37, 41]. We found that exclusively $w \mathrm{MelBr}$ couples were equally prone to interact as WT ones, with roughly the same proportion of samples showing duets. Mixed couples, where $w$ MelBr pairs with WT, were also able to interact albeit with apparently lower indexes (not statistically significant). In any case, it seems that $w \mathrm{MelBr}$ conserves mating behavior, communicating through specific acoustic signals and possibly promoting successful copulas.

Surprisingly, our data also revealed alternative harmonic interactions, occurring both under and above the putative 


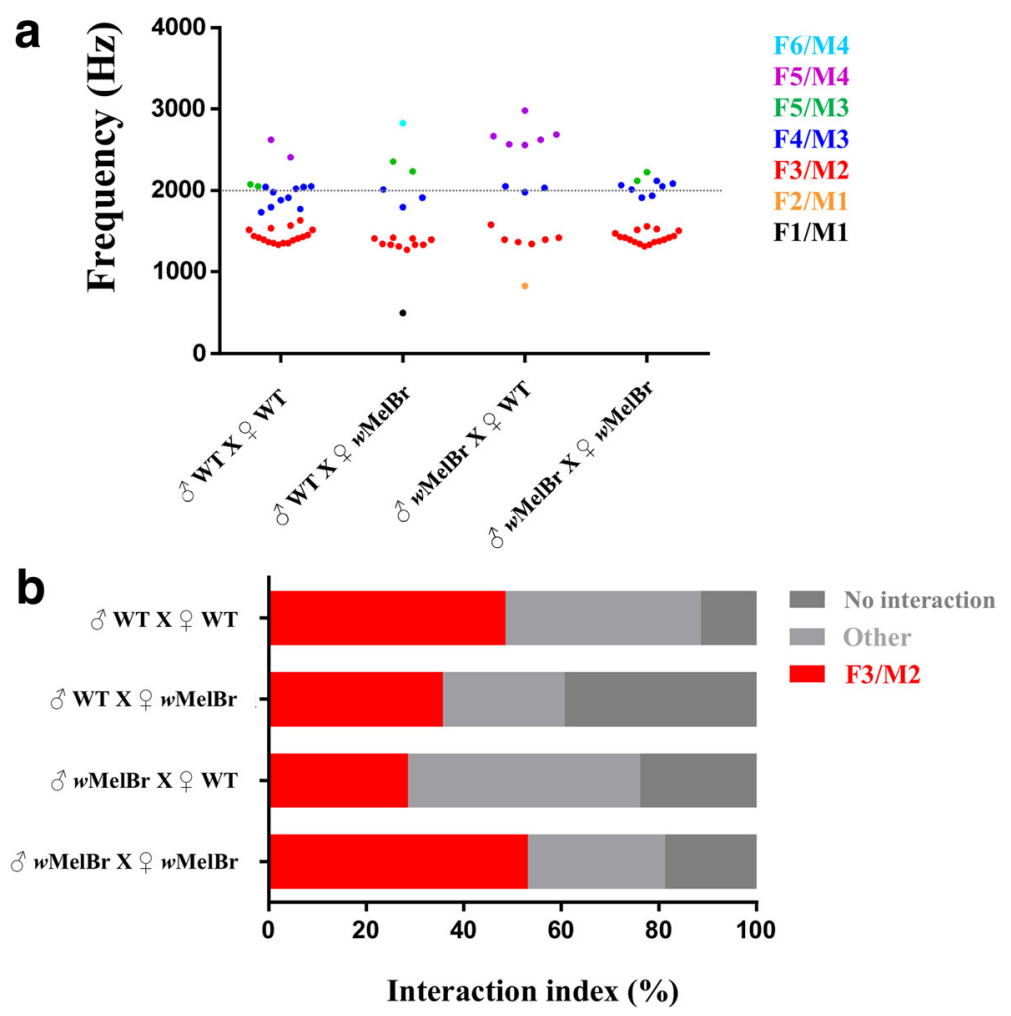

Fig. 3 Harmonic convergence of $w \mathrm{MelBr}$ and WT couples. a Distribution of converging samples per couple type. Converging samples were classified by color according to the harmonic frequencies involved in the acoustic interaction, where F/M represent female and male components. The dotted line at $2000 \mathrm{~Hz}$ depicts Ae. aegypti putative hearing threshold. b Relative contribution of F3/M2, alternative (other) and 'no interaction' samples

hearing threshold of $2000 \mathrm{~Hz}$ [37], with some few events reaching levels close to $3000 \mathrm{~Hz}$ (Fig. 3). We hypothesize that these interactions either have no biological significance or constitute important cues for acoustic communication between individuals. If the former is true, than interactions are merely artifacts promoted by odd relationships between two individuals' wing-beat frequencies. However, if the latter is true, than interactions not yet characterized might contribute to sexual communication by adding an extra level of complexity. This would also imply that electrophysiological recordings have not precisely defined the upper limit of Johnston organ (JO) sensitivity in Ae. aegypti and that this species may hear and communicate at frequencies a few hundred hertz higher than previously thought [37]. Alternatively, mosquitoes could be hearing not actual harmonic interactions but an output frequency obtained by the integration of male and female wing-beats $[48,49]$. In fact, mosquito hearing mechanisms are currently being revised, due largely to the advent of more comprehensive behavior and physiological audiograms (i.e. tuning curves) [48-50]. It has been reported that the auditory receptors within the JO are unequally represented and individually tuned to different frequency ranges [50]. Other sources of sensory input such as antennal and body hairs may also contribute to a broader range of auditory signals reaching the brain and thus augmenting sensitivity limits [48-50].

In addition, and despite the importance of our findings, recent evidence suggests that acoustic-related mating behavior includes aspects other than harmonic interactions [48, 49]. In species of Culex and Anopheles, males use acoustic distortion products to detect nearby flying females and to elicit rapid frequency modulation (RFM) of their wing-beats just prior to copula. This phenomenon appears to be essential for mating in both genus and may also exist in Aedes spp. Thus, one cannot discard the possibility that Wolbachia-harboring and WT males drive distinct RFM in response to their respective female flight tones, provoking a certain degree of assortative mating. In this context, the subtle effect found in the fundamental frequencies of $w \mathrm{MelBr}$ males and females (Fig. 1) could be differentially translated to acoustic distortions during an RFM response. However, recent evidence suggests that RFM could not to explain the reproductive isolation between sympatric species of the Anopheles complex [49]. Future studies are nonetheless necessary 
to address this point, as to whether the distortion products generated by Wolbachia-harboring males could provide any other means for locating and mating with its own variant type.

Regarding the use of Wolbachia for vector-borne disease control, our findings are greatly encouraging. The conserved pattern of mating acoustic signaling in individuals carrying the $w \mathrm{Mel}$ strain shall certainly contribute to their reproductive fitness and facilitate bacterial spread. Once released in the field, these individuals are expected to successfully mate and copulate with wild populations, transmitting the bacteria to the progeny. In subsequent generations, they should be able to breed not only with the wild-type but also with other $w$ Mel-harboring individuals, hence keeping the local bacteria load. Corroborating this idea, a mosquito population from Cairns (Australia) still holds $w$ Mel infection close to fixation after three years following initial field release [25]. It was also revealed a near perfect maternal transmission rate, as well as intact CI and PI (DENV-blocking), suggesting that Wolbachia-host interaction does not significantly change over a brief period of time $[25,51]$.

It is important to note that $w$ Mel's behavior phenotype may be restricted to this particular strain and genetic background, and should not be extrapolated to others without further investigation. It has already been shown that different Wolbachia strains elicit different bacteriahost interactions, hence different host behavioral, metabolic and physiological outcomes [35]. Relevant fitness traits and the particularly important PI phenotype have been measured for some strains, suggesting a delicate trade-off between both. For some strains with very strong PI, such as $w$ MelPop, the cost is so high for the host that it struggles to survive in the natural habitat $[52,53]$. In this case, it would not be a surprise to find that mating behavior is also disrupted, decreasing reproductive fitness. Conversely, strains like $w \mathrm{Mel}$ or $w \mathrm{Alb}$ are less harmful and often associated with milder fitness costs [35], yet still able to drive an effective PI. As our data suggest, mating behavior could be somewhat conserved for strains belonging to this category.

Finally, this work fully supports the current use of $w$ Melharboring lines to control the spread of dengue and other vector-borne diseases. Without significantly affecting some aspects of the acoustic signaling implied in mating success, as well as other critical traits $[23,26]$, these lines seem to be fit enough to promote the bacteria invasion in the wild, thus leading to reduced rates of disease transmission and a positive impact on local public health.

\section{Conclusions}

Our acoustic recordings and data analysis suggest that the $w$ Mel strain of Wolbachia is able to drive small sexdependent alterations on the fundamental flight tones of
Ae. aegypti. This effect, however, does not seem to prevent the formation of the 'so-called' harmonic duets between males and females. By preserving this important aspect of pre-copulatory behavior, Wolbachia-harboring individuals shall be fit enough to acoustically interact and successfully mate with wild variants in field release scenarios, thus contributing to bacteria dispersion and fixation over time.

\section{Additional files}

Additional file 1: Figure S1. Alternative acoustic interaction between the fourth harmonic frequency (F4) for a female (red) and the third harmonic frequency (M3) for a male (blue) of Ae. aegypti. (TIFF $1525 \mathrm{~kb}$ )

Additional file 2: Table S1. Descriptive statistics of the couple types. (TIFF 605 kb)

\section{Acknowledgements}

We would like to thank all members of Laboratório de Biologia Molecular de Insetos (LABIMI, IOC/Fiocruz) and Mosquitos Vetores: Endossimbiontes e Interação Patógeno Vetor (MV, CPqRR/FIOCRUZ) for continuous support and critical feedback during the course of this study. Our special thanks to Robson Costa da Silva, for expert technical assistance in experiment set up. We are also very grateful to the World Mosquito Program team, in particular to Professor Scott L. O'Neill for donating the original wMel line and for critically reviewing the manuscript.

\section{Funding}

This work was co-funded by CNPq, INCT and a grant to Monash University from the Foundation for the National Institutes of Health through the Vector-Based Transmission of Control: Discovery Research (VCTR) program of the Grand Challenges in Global Health Initiatives of the Bill and Melinda Gates Foundation.

\section{Availability of data and materials}

The datasets used and/or analyzed during the current study are available from the corresponding author upon reasonable request.

\section{Authors' contributions}

JSMG designed the study, carried out experiments, analyzed the data and drafted the manuscript. ASA carried out experiments, analyzed the data and reviewed the manuscript. CDO was responsible for colony maintenance and provision of eggs for the experiments. EPC performed statistical analysis and reviewed the manuscript. AJM provided expert knowledge and critical feedback during the experiments and manuscript preparation. RVB and LAM coordinated the work and critically reviewed the manuscript. All authors read and approved the final manuscript.

\section{Ethics approval and consent to participate}

Not applicable.

\section{Consent for publication}

Not applicable.

\section{Competing interests}

The authors declare that they have no competing interests.

\section{Publisher's Note}

Springer Nature remains neutral with regard to jurisdictional claims in published maps and institutional affiliations.

\section{Author details}

${ }^{1}$ Mosquitos Vetores: Endossimbiontes e Interação Patógeno-Vetor, Instituto de Pesquisas René Rachou, Fiocruz, Belo Horizonte, MG, Brazil. 'Laboratório de Biologia Molecular de Insetos, Instituto Oswaldo Cruz, Fiocruz, Rio de Janeiro, RJ, Brazil. ${ }^{3}$ Laboratório de Fisiologia e Controle de Artrópodes Vetores, Instituto Oswaldo Cruz, Fiocruz, Rio de Janeiro, RJ, Brazil. ${ }^{4}$ Instituto Nacional de Ciência e Tecnologia em Entomologia Molecular (INCT-EM)/ CNPq, Rio de Janeiro, Brazil. 


\section{Received: 29 September 2017 Accepted: 2 February 2018}

\section{Published online: 22 February 2018}

\section{References}

1. Bhatt S, Gething PW, Brady OJ, Messina JP, Farlow AW, Moyes CL, et al. The global distribution and burden of dengue. Nature. 2013;496:504-7.

2. Leparc-Goffart I, Nougairede A, Cassadou S, Prat C, de Lamballerie X. Chikungunya in the Americas. Lancet. 2014;383:514.

3. Morrison TE. Reemergence of chikungunya virus. J Virol. 2014:88:11644-7.

4. Jentes ES, Poumerol G, Gershman MD, Hill DR, Lemarchand J, Lewis RF, et al. The revised global yellow fever risk map and recommendations for vaccination, 2010: consensus of the informal WHO working group on geographic risk for yellow fever. Lancet Infect Dis. 2011;11:622-32.

5. Enserink M. Infectious diseases. An obscure mosquito-borne disease goes global. Science. 2015;350:1012-3.

6. Brady OJ, Gething PW, Bhatt S, Messina JP, Brownstein JS, Hoen AG, et al. Refining the global spatial limits of dengue virus transmission by evidencebased consensus. PLoS Negl Trop Dis. 2012;6:e1760.

7. Weaver SC. Arrival of chikungunya virus in the new world: prospects for spread and impact on public health. PLoS Negl Trop Dis. 2014;8:e2921.

8. Wahid B, Ali A, Rafique S, Idrees M. Global expansion of chikungunya virus: mapping the 64-year history. Int J Infect Dis. 2017:58:69-76.

9. Nugent EK, Nugent AK, Nugent R, Nugent K. Zika virus: epidemiology, pathogenesis and human disease. Am J Med Sci. 2017:353:466-73.

10. Mayer SV, Tesh RB, Vasilakis N. The emergence of arthropod-borne viral diseases: a global prospective on dengue, chikungunya and Zika fevers. Acta Trop. 2017;16:155-63.

11. Garske T, Van Kerkhove MD, Yactayo S, Ronveaux O, Lewis RF, Staples JE, et al. Yellow fever in Africa: estimating the burden of disease and impact of mass vaccination from outbreak and serological data. PLoS Med. 2014;11:e1001638.

12. Carvalho FD, Moreira LA. Why is Aedes aegypti Linnaeus so successful as a species? Neotrop Entomol. 2017:46:243-55.

13. David MR, Lourenço-de-Oliveira R, De Freitas RM. Container productivity, daily survival rates and dispersal of Aedes aegypti mosquitoes in a high income denque epidemic neighbourhood of Rio de Janeiro: presumed influence of differential urban structure on mosquito biology. Mem Inst Oswaldo Cruz. 2009:104:927-32.

14. McBride CS, Baier F, Omondi AB, Spitzer AS, Lutomiah J, Sang R, et al. Evolution of mosquito preference for humans linked to an odorant receptor. Nature. 2014;515:222-7.

15. Kraemer MU, Sinka ME, Duda KA, Mylne AQ, Shearer FM, Barker CM, et al. The global distribution of the arbovirus vectors Aedes aegypti and Ae. albopictus. eLife. 2015;4:e08347.

16. Campbell LP, Luther C, Moo-Llanes D, Ramsey JM, Danis-Lozano R, Peterson AT. Climate change influences on global vector distributions for dengue and chikungunya viruses. Phil Trans Roy Soc B. 2015;370:20140135.

17. Valença MA, Marteis LS, Steffler LM, Silva AM, Santos RL. Dynamics and characterization of Aedes aegypti (L.) (Diptera: Culicidae) key breeding sites. Neotrop Entomol. 2013;42:311-6.

18. Ritchie SA, Devine GJ. Confusion, knock-down and kill of Aedes aegypti using metofluthrin in domestic settings: a powerful tool to prevent dengue transmission? Parasit Vectors. 2013;6:262.

19. Abad-Franch F, Zamora-Perea E, Ferraz G, Padilla-Torres SD, Luz SL. Mosquito-disseminated pyriproxyfen yields high breeding-site coverage and boosts juvenile mosquito mortality at the neighborhood scale. PLoS Negl Trop Dis. 2015;9:1-17.

20. Valle D, Belinato TA, Martins AJ. Controle químico de Aedes aegypti, resistência a inseticidas e alternativas. In: Valle D, Pimenta DN, da Cunha RV, editors. Denque: teorias e práticas. Rio de Janeiro: Fiocruz; 2015. p. 93-126.

21. Linss JG, Brito LP, Garcia GA, Araki AS, Bruno RV, Lima JB, et al. Distribution and dissemination of the Val1016lle and Phe1534Cys kdr mutations in Aedes aegypti Brazilian natural populations. Parasit Vectors. 2014;7:25.

22. Maciel-de-Freitas R, Avendanho FC, Santos R, Sylvestre G, Araújo SC, Lima JB, et al. Undesirable consequences of insecticide resistance following Aedes aegypti control activities due to a dengue outbreak. PLoS One. 2014;9:e92424.

23. Walker $\mathrm{T}$, Johnson $\mathrm{PH}$, Moreira LA, Iturbe-Ormaetxe I, Frentiu F, McMeniman CJ, et al. The WMel Wolbachia strain blocks dengue and invades caged Aedes aegypti populations. Nature. 2011:476:450-3.

24. Hoffmann AA, Montgomery BL, Popovici J, Iturbe-Ormaetxe I, Johnson PH, Muzzi F, et al. Successful establishment of Wolbachia in Aedes populations to suppress dengue transmission. Nature. 2011;476:454-7.
25. Hoffmann AA, Iturbe-Ormaetxe I, Callahan AG, Phillips BL, Billington K, Axford JK, et al. Stability of the wMel Wolbachia infection following invasion into Aedes aegypti populations. PLoS Negl Trop Dis. 2014;8:e3115.

26. Dutra HL, Dos Santos LM, Caragata EP, Silva JB, Villela DA, Maciel-de-Freitas $R$, Moreira LA. From lab to field: the influence of urban landscapes on the invasive potential of Wolbachia in Brazilian Aedes aegypti mosquitoes. PLoS Negl Trop Dis. 2015;9:e0003689.

27. Zug R, Hammerstein P. Still a host of hosts for Wolbachia: analysis of recent data suggests that $40 \%$ of terrestrial arthropod species are infected. PLoS One. 2012;7:e38544.

28. Werren JH, Baldo L, Clark ME. Wolbachia: master manipulators of invertebrate biology. Nat Rev Microbiol. 2008;6:741-51.

29. McMeniman CJ, Lane RV, Cass BN, Fong AW, Sidhu M, Wang YF, et al. Stable introduction of a life-shortening Wolbachia infection into the mosquito Aedes aegypti. Science. 2009;323:141-4.

30. Moreira LA, Iturbe-Ormaetxe I, Jeffery JA, Lu G, Pyke AT, Hedges LM, et al. A Wolbachia symbiont in Aedes aegypti limits infection with dengue, chikungunya, and Plasmodium. Cell. 2009;139:1268-78.

31. Dutra HL, Rocha MN, Dias FB, Mansur SB, Caragata EP, Moreira LA. Wolbachia blocks currently circulating Zika virus isolates in Brazilian Aedes aegypti mosquitoes. Cell Host Microbe. 2016;19:771-4.

32. McMeniman CJ, O'Neill SL. A virulent Wolbachia infection decreases the viability of the dengue vector Aedes aegypti during periods of embryonic quiescence. PLoS Negl Trop Dis. 2010;4:e748.

33. Turley AP, Moreira LA, O'Neill SL, McGraw EA. Wolbachia infection reduces blood-feeding success in the dengue fever mosquito, Aedes aegypti. PLoS Negl Trop Dis. 2009;3:e516.

34. Evans O, Caragata EP, McMeniman CJ, Woolfit M, Green DC, Williams CR, et al. Increased locomotor activity and metabolism of Aedes aegypti infected with a life-shortening strain of Wolbachia pipientis. J Exp Biol. 2009;212:1436-41.

35. Caragata EP, Dutra HL, Moreira LA. Exploiting intimate relationships: controlling mosquito-transmitted disease with Wolbachia. Trends Parasitol. 2016:32:207-18.

36. Gibson G, Russell I. Flying in tune: sexual recognition in mosquitoes. Curr Biol. 2006;16:1311-6.

37. Cator LJ, Arthur BJ, Harrington LC, Hoy RR. Harmonic convergence in the love songs of the denque vector mosquito. Science. 2009;323:1077-9.

38. Cator LJ, Ng'Habi KR, Hoy RR, Harrington LC. Sizing up a mate: variation in production and response to acoustic signals in Anopheles gambiae. Behav Ecol. 2010;21:1033-9.

39. Warren B, Gibson G, Russell IJ. Sex recognition through midflight mating duets in Culex mosquitoes is mediated by acoustic distortion. Curr Biol. 2009;19:485-91.

40. Pennetier C, Warren B, Dabiré KR, Russell IJ, Gibson G. "Singing on the wing" as a mechanism for species recognition in the malarial mosquito Anopheles gambiae. Curr Biol. 2010;20:131-6.

41. Cator LJ, Harrington LC. The harmonic convergence of fathers predicts the mating success of sons in Aedes aegypti. Anim Behav. 2011;82:627-33.

42. Clements AN. The biology of mosquitoes. Vol. 2. Sensory reception and behaviour. New York: CABI Publishing; 1999.

43. Gorczyca M, Hall JC. The INSECTAVOX, an integrated device for recording and amplifying courtship songs of Drosophila. Dros Inf Serv. 1987;66:157-60.

44. Stirnemann RL, Potter MA, Butler D, Minot EO. Acoustic differences enable sex discrimination in Ma'oma'o (Gymnomyza samoensis), a species with high sexual morphological overlap. Wilson J Ornithol. 2015;127:376-86.

45. Wishart G, Riordan DF. Flight responses to various sounds by adult males of Aedes aegypti (L.) (Diptera: Culicidae). Can Entomol. 1959;91:181-91.

46. Charlwood JD, Jones MDR. Mating behaviour in the mosquito, Anopheles gambiae s.l. I. Close range and contact behaviour. Physiol Entomol. 1979;4:111-20

47. Belton P. Attraction of male mosquitoes to sound. J Am Mosq Control Assoc. 1994;10:297-301.

48. Simões PMV, Ingham RA, Gibson G, Russell IJ. A role for acoustic distortion in novel rapid frequency modulation behaviour in free-flying male mosquitoes. J Exp Biol. 2016;219:2039-47.

49. Simões PMV, Gibson G, Russell IJ. Pre-copula acoustic behaviour of males in the malarial mosquitoes Anopheles coluzzii and Anopheles gambiae s.s. does not contribute to reproductive isolation. J Exp Biol. 2017;220:379-85.

50. Lapshin DN, Vorontsov DD. Frequency tuning of individual auditory receptors in female mosquitoes (Diptera, Culicidae). J Insect Physiol. 2013;59:828-39.

51. Frentiu FD, Zakir T, Walker T, Popovici J, Pyke AT, van den Hurk A, et al. Limited dengue virus replication in field-collected Aedes aegypti mosquitoes infected with Wolbachia. PLoS Negl Trop Dis. 2014;8:e2688. 
52. Yeap HL, Axford JK, Popovici J, Endersby NM, Iturbe-Ormaetxe I, Ritchie SA et al. Assessing quality of life-shortening Wolbachia-infected Aedes aegypti mosquitoes in the field based on capture rates and morphometric assessments. Parasit Vectors. 2014;7:58.

53. Ritchie SA, Townsend M, Paton CJ, Callahan AG, Hoffmann AA. Application of wMelPop Wolbachia strain to crash local populations of Aedes aegypti. PLoS Negl Trop Dis. 2015;9:e0003930.

Submit your next manuscript to BioMed Central and we will help you at every step:

- We accept pre-submission inquiries

- Our selector tool helps you to find the most relevant journal

- We provide round the clock customer support

- Convenient online submission

- Thorough peer review

- Inclusion in PubMed and all major indexing services

- Maximum visibility for your research

Submit your manuscript at www.biomedcentral.com/submit
Biomed Central 